\title{
Sobre los materiales de encapsulado para electrodos selectivos de iones
}

\section{On the encapsulation materials for ion-selective electrodes}

\author{
Josefina M. Silveyra ${ }^{1,2}$, Andrea Ureña ${ }^{1,2}$, \\ Juan Manuel Conde Garrido ${ }^{1,2}$
}

\author{
${ }^{1}$ Universidad de Buenos Aires, Facultad de Ingeniería, Laboratorio de Sólidos Amorfos, Av. Paseo Colón 850, (1063), \\ Buenos Aires, Buenos Aires, Argentina. \\ ${ }^{2}$ CONICET - Universidad de Buenos Aires, Instituto de Tecnologías y Ciencias de la Ingeniería "Hilario Fernadez Long" \\ (INTECIN), Buenos Aires, Buenos Aires, Argentina. \\ e-mail: jmcondegarrido@fi.uba.ar
}

\section{RESUMEN}

Los electrodos selectivos de iones (ISEs) con membranas de vidrios calcogenuros son sensores capaces de detectar iones de metales pesados en soluciones electrolíticas. Su uso in-situ y repetido plantea grandes desafíos a la selección del material para encapsular la membrana sensible. La falla en el encapsulado inutiliza al sensor, que debe ser descartado. Sin embargo, no existe hasta ahora estudios reportados sobre este componente crítico de los sensores. El material encapsulante debe aislar al contacto eléctrico de la solución circundante, tener un bajo nivel de absorción de la solución y de los iones en solución, así como mantener sus propiedades invariables por largos períodos de tiempo. Los requisitos del material dependerán también de la geometría de la membrana, ya sea en bulk o en forma de película delgada. Con el fin de facilitar nuevas investigaciones en la temática de los ISEs, estudiamos cinco materiales encapsulantes disponibles comercialmente en la región latinoamericana (Poxipol transparente, Vaicel, Wilpox APV 3C, Wilpox Doming P-5 y Araldite CY-248 + HY-956). Analizamos los requisitos de estabilidad y confiabilidad evaluando las siguientes propiedades: viscosidad, tiempo de curado, dureza, calidad superficial, formación de burbujas, respuesta al pulido mecánico, reacción con pintura de Ag, efecto del agua y de soluciones de limpieza y almacenamiento. Encontramos que, tomando los cuidados necesarios, las resinas epoxi Wilpox APV 3C y Araldite CY-248 + HY-956 son las más idóneas para encapsular membranas de vidrios calcogenuros en bulk, mientras que la resina epoxi Wilpox APV 3C y la resina acrílica Vaicel resultan ser las mejores candidatas para el caso de las membranas en forma de película delgada.

Palabras clave: encapsulado, membranas, electrodos selectivos de iones, vidrios calcogenuros.

\begin{abstract}
Ion-selective electrodes (ISEs) built with chalcogenide glass membranes are sensors capable of detecting heavy metal ions in electrolytic solutions. Using ISEs in-situ and repeatedly can be challenging not only to the sensitive membrane, but to the material used to encapsulate the electrical contact between the membrane and the metallic wire. If the encapsulation fails, the sensor must be discarded. Despite how critical these issues are on this sensor component, no studies on the requirements and selection of the encapsulation material have been reported so far. This material must isolate the electrical contact of the surrounding solution, have a low level of absorption of the solution and the ions in the solution, as well as keep its properties stable in the long-term. The material requirements will also depend on the geometry of the membrane, either bulk or thin film. To stimulate further research and development in the field of ISEs, we investigated five encapsulating materials commercially available in the Latin American region (transparent Poxipol, Vaicel, Wilpox APV 3C, Wilpox Doming P-5 and Araldite CY-248 + HY-956). We analyzed the encapsulation requirements to enable stable and reliable ISEs by evaluating the following properties: viscosity, curing time, hardness, surface quality, formation of bubbles, effect of mechanical polishing, reaction to Ag paint, effect of water, and cleaning and storage solutions. We found that, under
\end{abstract}


the proper conditions, Wilpox APV 3C and Araldite CY-248 + HY-956 epoxy resins are the most suitable materials to encapsulate bulk chalcogenide glass membranes, while Wilpox APV 3C epoxy resin and Vaicel acrylic resin turn out to be the best candidates for thin film membranes.

Keywords: encapsulation, membranes, ion-selective electrodes, chalcogenide glasses.

\section{INTRODUCCIÓN}

Los electrodos selectivos de iones (ion-selective electrodes, ISEs) son el grupo más grande de los sensores potenciométricos y se utilizan en diversos campos, tales como en la industria y el monitoreo ambiental de aguas [1].

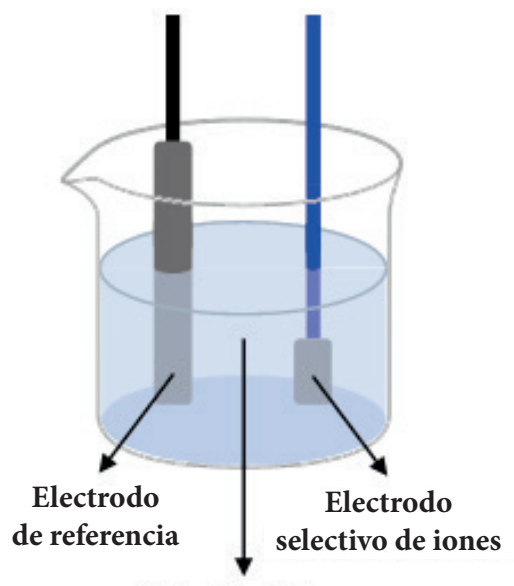

Solución iónica

Figura 1: Sistema de medición electro-química.

Cuando un ISE es sumergido en una solución junto con un electrodo de referencia, la diferencia de potencial que se establece entre ambos está directamente relacionada con la concentración de algún ion específico mediante la ley de Nernst [2] (ver esquema del sistema de medición en Figura 1). La membrana del ISE en contacto con la solución juega un rol clave en el sensor, ya que diferentes materiales brindan una sensibilidad distinta a distintos iones. Los ISEs permiten medir en tiempo real e in-situ y detectan únicamente especies ionizadas. Esto último es esencial para el control de contaminación industrial y ambiental, ya que es precisamente en el estado iónico que las especies son más nocivas para los organismos [3].

Un grupo importante de membranas es el basado en vidrios calcogenuros, habiendo sido reportado por primera vez en 1969 por TRACHTENBERG y BAKER [4]. Existe una amplia familia de sistemas de vidrios calcogenuros con un amplio espectro de propiedades físicas, químicas y electroquímicas [2]. Estos materiales tienen varias ventajas: alta selectividad, excelente durabilidad y buena estabilidad química [5]. Además, las membranas de vidrios calcogenuros permiten fabricar sensores de contacto sólido (solid-contact sensors, es decir, sin solución líquida interna) e incluso ISEs miniaturizados (conocidos como $\mu$ ISEs [6]) y arreglos multisensores ("lengua electrónica" [7]), ya que es posible sintetizar los vidrios en forma de películas delgadas.

Debido a que los ISEs trabajan en soluciones electrolíticas, estos deben ser encapsulados de manera tal que únicamente la membrana sensible quede expuesta a la solución, mientras que el contacto eléctrico permanezca aislado. La utilización in-situ y repetida de los ISEs, plantea grandes desafíos para el material de encapsulado: tener buena adherencia a la membrana y al alambre conductor, mantener sus propiedades aislantes durante largos períodos de tiempo y contar con un bajo nivel de absorción de los iones en solución. La elección del material (comúnmente alguna resina epoxi o silicona [8]) y la técnica de encapsulamiento van a estar determinados por el diseño y geometría del dispositivo sensor, así como por sus condiciones de trabajo. Históricamente, las investigaciones se han focalizado principalmente en el desempeño de distintas aleaciones como membranas selecticas de iones, con poca atención al material para encapsularlas. De hecho, no pareciera haber una receta universal para el proceso de encapsulado, que suele ser una tarea manual, lenta y tediosa, con un alto grado de fallas $[9,10]$. Desafortunadamente, las fallas generalmente se detectan demasiado tarde, con el electrodo completamente armado y los datos que éste aporte deben ser descartados.

Medio siglo después de la primera publicación sobre ISEs basados en vidrios calcogenuros, todavía continúa el interés en el desarrollo y mejora de las membranas calcogenuras selectivas de iones [2]. Pero el encapsulado de las membranas 
selectivas de iones resulta crítico para la correcta caracterización del sensor, así como para su estabilidad y confiabilidad a largo plazo. En este trabajo, nuestro objetivo fue identificar el o los materiales más idóneos para encapsular membranas de electrodos selectivos de iones, tanto en bulk como en forma de películas delgadas. Para ello, evaluamos propiedades químicas y mecánicas relevantes para los ISEs de materiales para encapsular disponibles comercialmente en la región, con el objetivo de facilitar nuevas investigaciones en la temática.

\section{MATERIALES Y MÉTODOS}

Analizamos cinco materiales disponibles comercialmente en la región latinoamericana, en vistas a ser utilizados como material de encapsulado de ISEs de contacto sólido basados en vidrios calcogenuros, tanto en bulk (Figura 2) como en forma de película delgada (Figura 3).

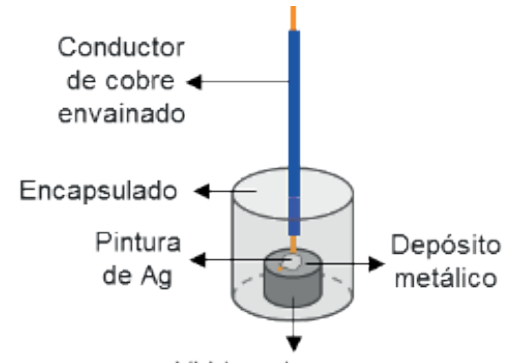

Vidrio calcogenuro
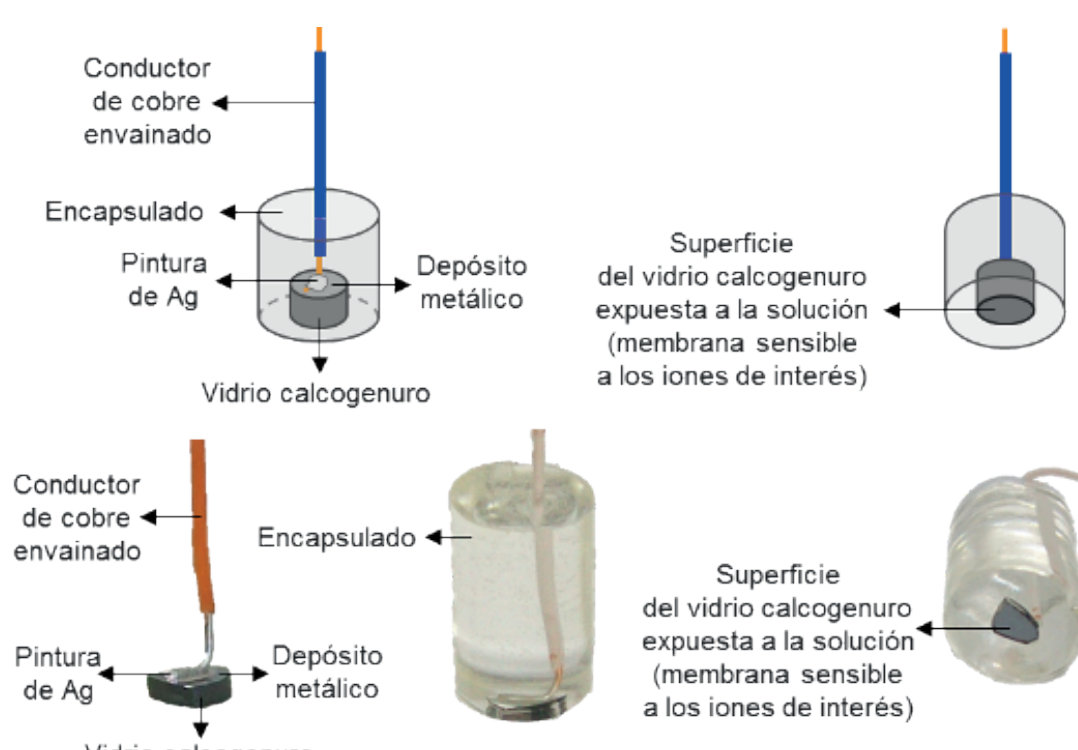

(membrana sensible

a los iones de interés)

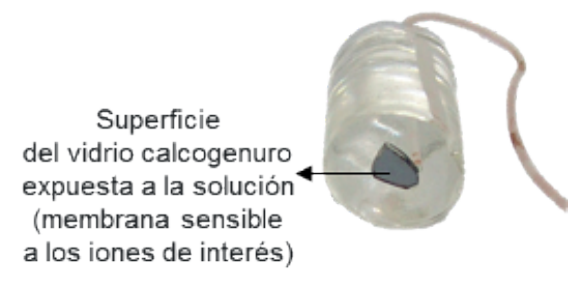

a los iones de interés)

Figura 2: Esquemas (arriba) y fotografías (abajo) del electrodo selectivo de iones de contacto sólido con vidrio calcogenuro en bulk. El encapsulado cilíndrico es de $\sim 1 \mathrm{~cm}$ de diámetro y $\sim 2 \mathrm{~cm}$ de alto.
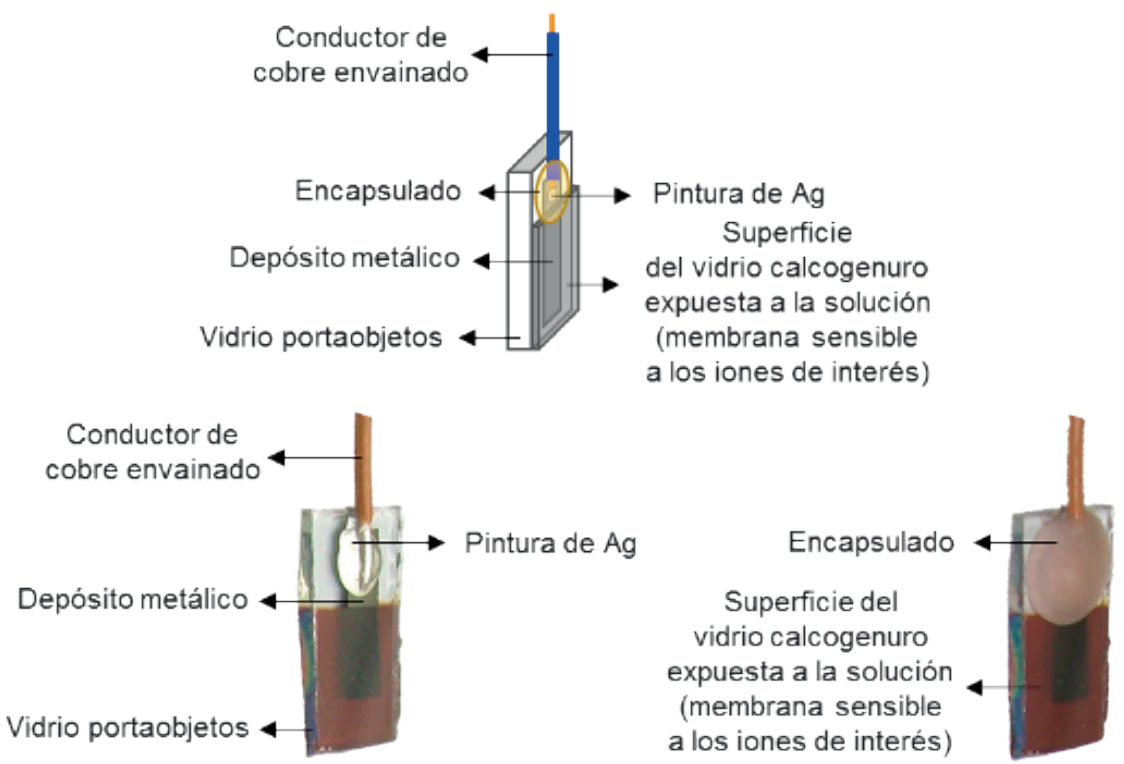

Figura 3: Esquema (arriba) y fotografías (abajo) del electrodo selectivo de iones de contacto sólido con película delgada de vidrio calcogenuro. El vidrio portaobjetos es de $\sim 0,5 \mathrm{~cm}$ de ancho y $\sim 1,5 \mathrm{~cm}$ de alto. 
Los cinco materiales encapsulantes analizados se preparan a partir de dos componentes y son autocurables:

1) Poxipol®: Resina epoxi (transparente) y amina terciaria. Relación 1 a 1 en volumen.

2) Vaicel® (acrílico): Polímero (polvo) y monómero (líquido). Relación no indicada por el fabricante.

3) Wilpox® APV 3C: Resina epoxi (5007-A) y endurecedor (5007-B). Relación 2 a 1 en volumen.

4) Wilpox® Doming P-5: Resina epoxi (5008-A) y endurecedor (5008-B). Relación 2 a 1 en volumen.

5) Araldite®: Resina epoxi (CY-248) y endurecedor (HY-956). Relación 5 a 1 en peso.

En vistas a su aplicación como materiales para encapsular membranas selectivas de iones, analizamos las siguientes propiedades:

1) Viscosidad de la mezcla. De ella depende la facilidad de conformación del encapsulado. Utilizamos el método de caída de bola para medir la viscosidad a $20{ }^{\circ} \mathrm{C}$ y $\sim 5$ minutos después de comenzar a mezclar los dos componentes. No fue posible medir la viscosidad del Poxipol ni del Vaicel, debido a su muy rápido aumento con el tiempo.

2) Tiempo de curado a $25^{\circ} \mathrm{C}$. Para conocer el tiempo de preparación del encapsulado.

3) Dureza alcanzada después del curado. Utilizamos un durómetro portátil marca Petri modelo HL10 con un dispositivo de impacto tipo D (método Leeb).

4) Calidad superficial. Se prefiere una superficie lisa para evitar turbulencias y/o zonas de vórtices en la solución en agitación, ya que la concentración de iones de la solución podría no ser homogénea. Además, si el material encapsulante absorbiera solución (con o sin iones), la cantidad absorbida aumentaría con la rugosidad del encapsulado.

5) Predisposición a la formación de burbujas. Se prefiere que la presencia de burbujas en el encapsulado sea mínima, ya que estas podrían disminuir la adhesión con el vidrio calcogenuros y/o el alambre conductor.

6) Respuesta al pulido mecánico. Dado que la membrana del ISE puede bloquearse o agotarse debido a factores tales como la contaminación, disolución, oxidación, etc., es recomendable pulir su superficie para regenerarla periódicamente $[11,12]$. Es imprescindible contar con un encapsulado que, al ser sometido a tensiones mecánicas durante el pulido, no pierda la adhesión con el vidrio calcogenuro. Sometimos vidrios calcogenuros en bulk encapsulados con los distintos materiales a un pulido mecánico manual con lijas al agua de granulometría 600 .

7) Efecto del material encapsulante sobre pintura de Ag seca. Utilizamos pintura de Ag para adherir el alambre conductor al depósito metálico sobre el vidrio calcogenuro. Es crítico que este contacto no se deteriore al entrar en contacto con el material encapsulante.

8) Comportamiento tras la inmersión en agua, dado que el ISE detecta iones de metales pesados en soluciones acuosas. La absorción de agua modifica las propiedades del material encapsulante (aumenta el volumen del material encapsulante -lo que podría deteriorar la adhesión- aumenta la conductividad eléctrica -deteriorándose las propiedades aislantes-, etc.). Además, si junto con el agua, el material absorbiera iones, se modificaría la concentración iónica de la solución (si esta fuera muy baja) y podría contaminar futuras soluciones a detectar al liberar los iones absorbidos previamente.

9) Comportamiento frente a la inmersión en etanol y acetona, por ser posibles soluciones de limpieza de contaminación orgánica de la membrana sensible.

10) Comportamiento frente a la inmersión en $\operatorname{AgNO}_{3}\left(10^{-2} \mathrm{M}\right)$, por ser una posible solución de almacenamiento de ISEs, en los que la conductividad de la membrana sensible está basada en la movilidad de iones de Ag [13, 14].

\section{RESULTADOS}

En las Figuras 4 a 11 y en la Tabla 1 se reportan los resultados de las observaciones y mediciones realizadas sobre los cinco materiales encapsulantes evaluados. 


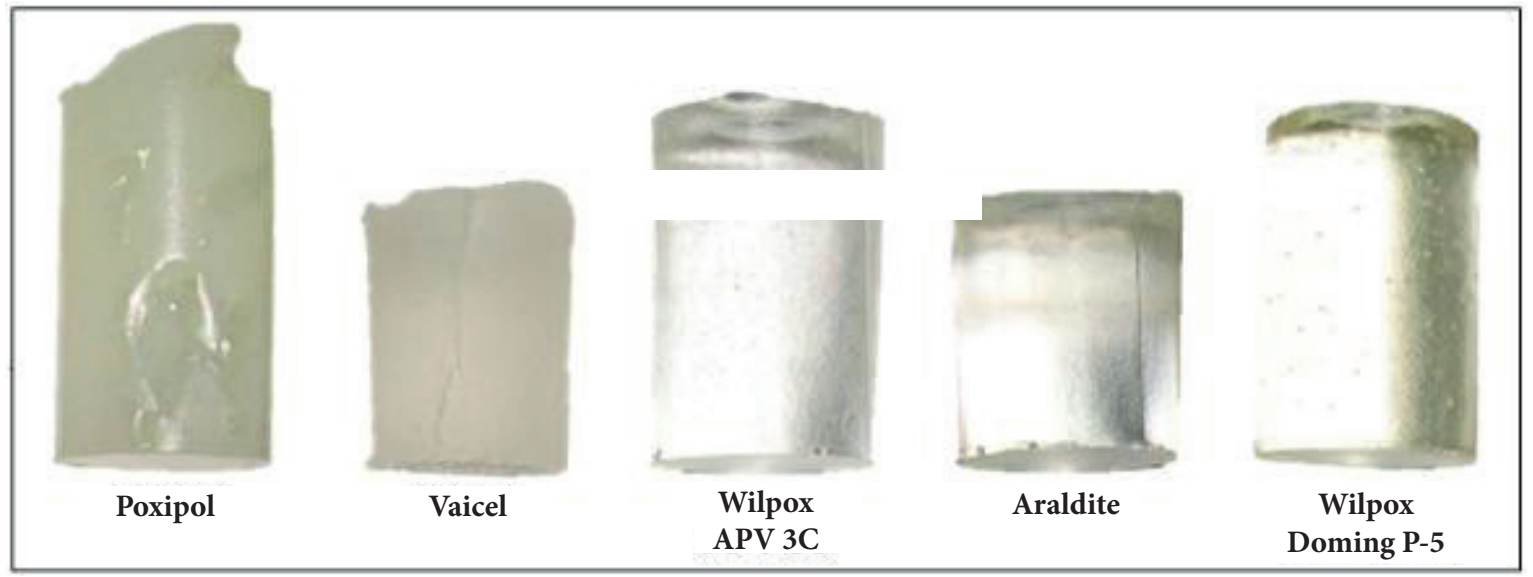

Figura 4: Materiales encapsulantes en geometría cilíndrica de $\sim 1 \mathrm{~cm}$ de diámetro.

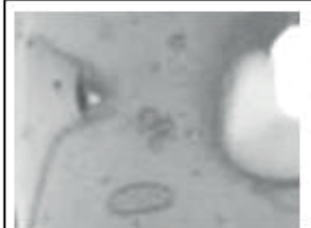

Poxipol

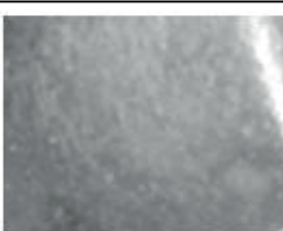

Vaicel

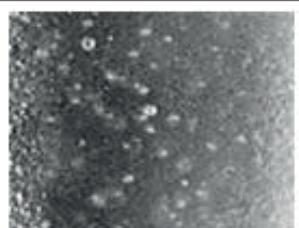

Wilpox

APV 3C

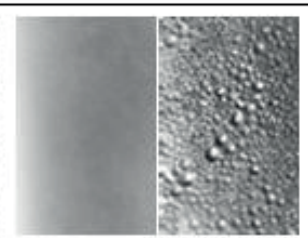

Araldite

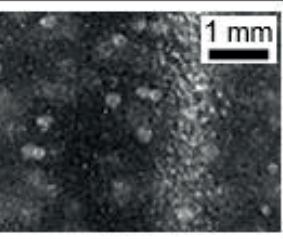

Wilpox

Doming P-5

Figura 5: Materiales encapsulantes. En el caso del Araldite, se exhibe antes (izquierda) y después (derecha) de haber sido expuesto a un ambiente húmedo durante 1 día.

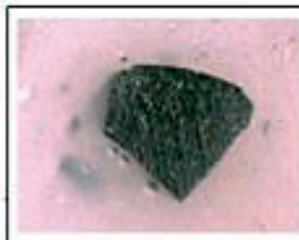

Poxipol

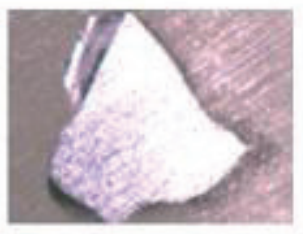

Vaicel

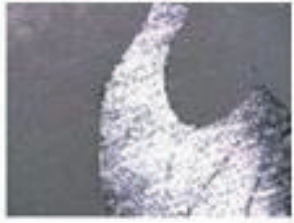

Wilpox APV 3C

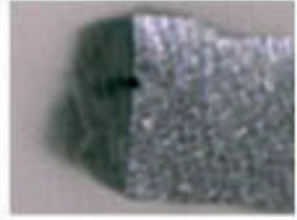

Araldite

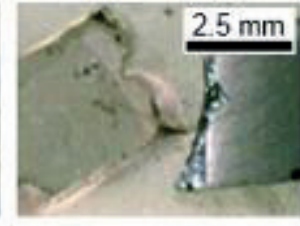

Wilpox

Doming P-5

Figura 6: Vidrios calcogenuros en bulk encapsulados y lijados. En el caso del Wilpox Doming P-5, el vidrio se salió del encapsulamiento durante el pulido.

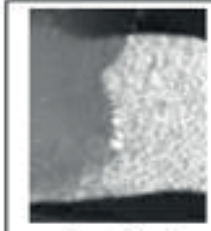

Poxipol

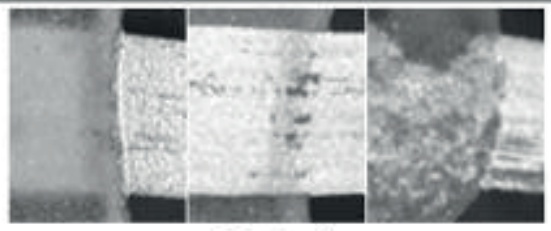

Vaicel

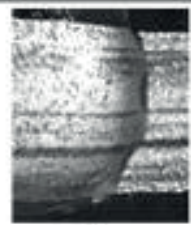

Wilpox APV 3C

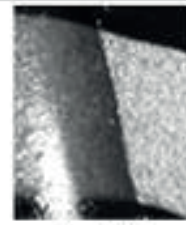

Araldite

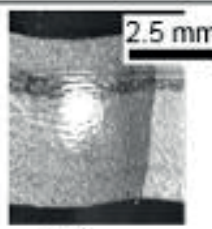

Wilpox

Doming P-5

Figura 7: Materiales encapsulantes sobre una pincelada seca de pintura de plata en un vidrio portaobjetos horizontal. En el caso del Vaicel, se presenta también la fotografía obtenida desde abajo del vidrio (centro) y desde arriba, pero habiéndose curado el material con el vidrio portaobjetos a $45^{\circ}$ (derecha). El Vaicel es el único de los materiales estudiados que disuelve y corre la pintura de plata. 

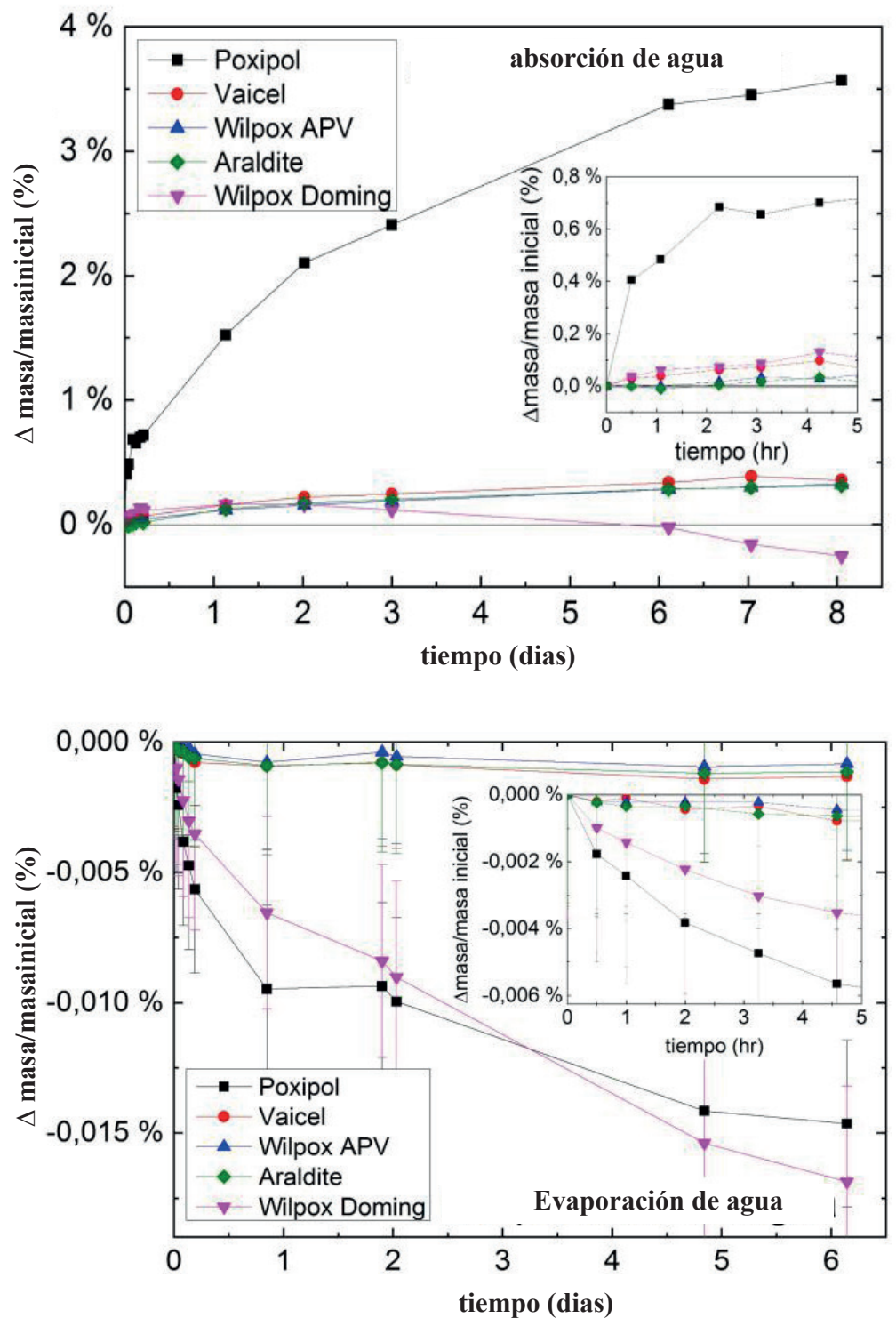

Figura 8: Respuesta de los materiales encapsulantes (con geometría cilíndrica) frente al agua. Arriba: Absorción de agua. Abajo: Evaporación tras 8 días de inmersión en agua. Las mediciones de variación de masa tienen un error del orden del $0,003 \%$ (las barras de error no se llegan a apreciar en el gráfico de absorción de agua).

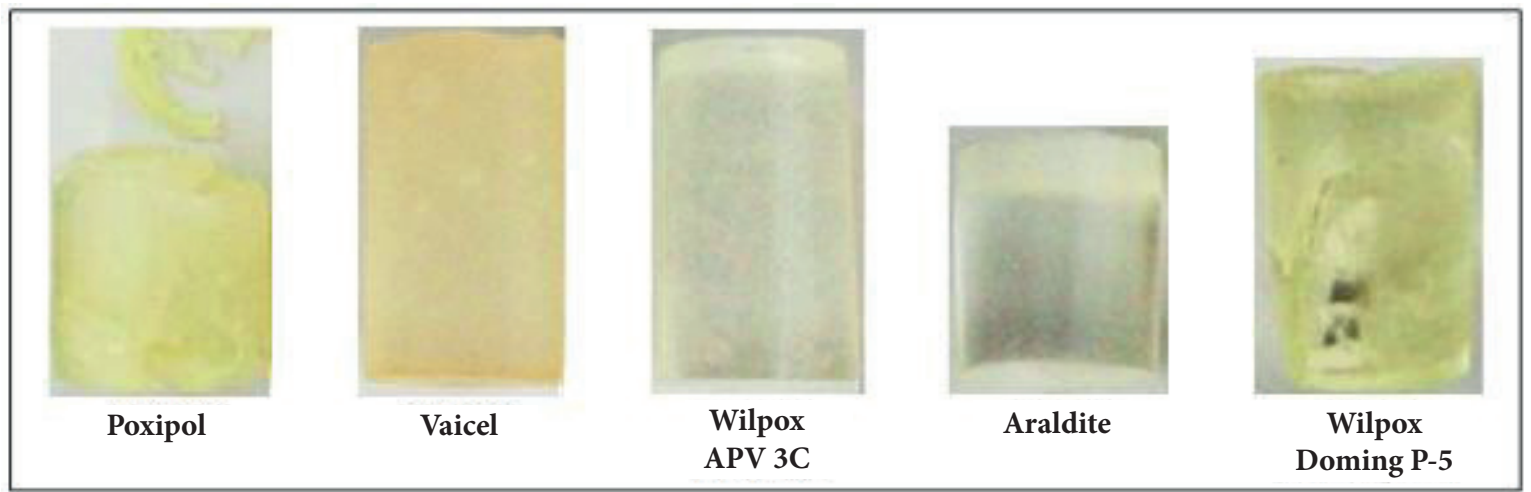

Figura 9: Materiales encapsulantes en geometría cilíndrica tras 1 día de inmersión en etanol. 


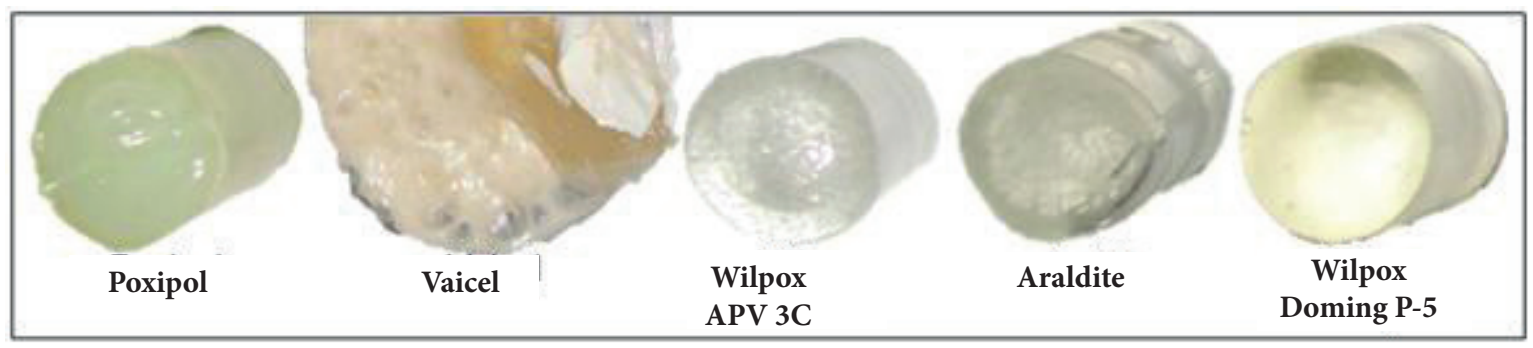

Figura 10: Materiales encapsulantes en geometría cilíndrica tras 1 día de inmersión en acetona. El cilindro de Vaicel perdió la forma inicial.

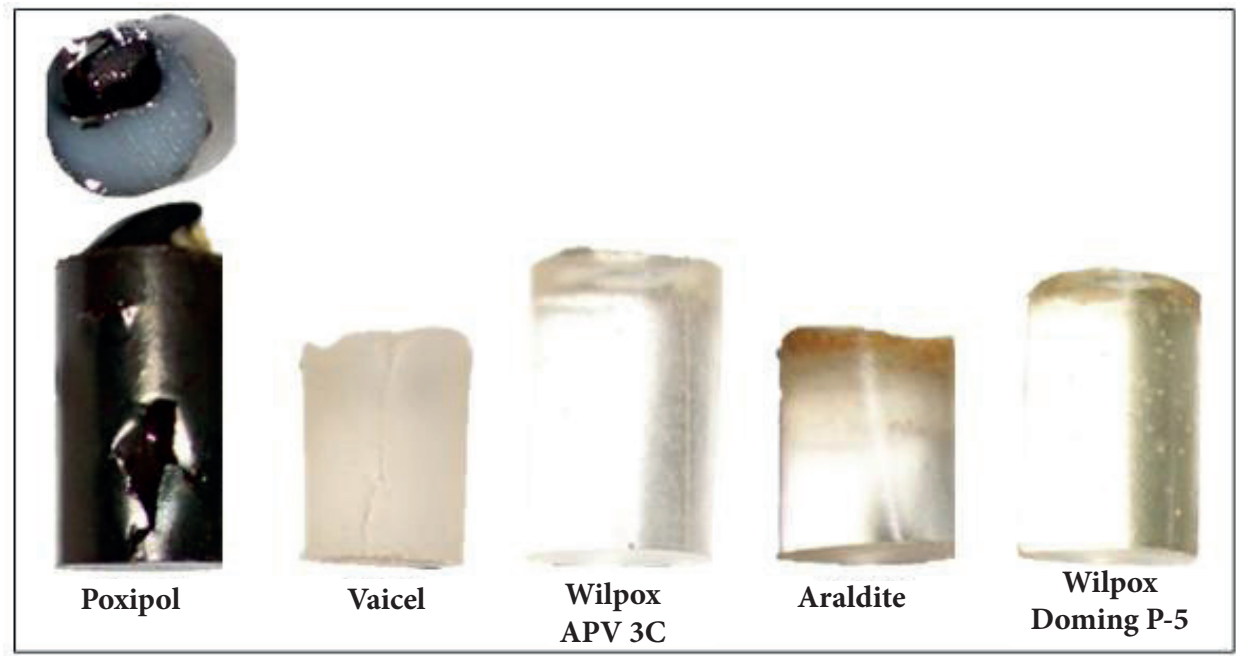

Figura 11: Materiales encapsulantes en geometría cilíndrica tras 1 día de inmersión en $\operatorname{AgNO}_{3}\left(10^{-2} \mathrm{M}\right)$. En el caso del Poxipol, se exhibe también un corte transversal del cilindro (arriba).

Tabla 1: Propiedades de los materiales encapsulantes.

\begin{tabular}{|c|c|c|c|c|c|}
\hline $\begin{array}{c}\text { MATERIAL } \\
\text { ENCAPSULANTE }\end{array}$ & POXIPOL & VAICEL & $\begin{array}{l}\text { WILPOX } \\
\text { APV 3C }\end{array}$ & ARALDITE & $\begin{array}{c}\text { WILPOX } \\
\text { DOMING P-5 }\end{array}$ \\
\hline $\begin{array}{l}\text { Viscosidad de la mezcla } \\
{[\mathrm{kg} /(\mathrm{m} \times \mathrm{s})](\text { error: } 5 \%)}\end{array}$ & $\begin{array}{l}\text { Muy viscoso. } \\
\text { Aumenta muy } \\
\text { rápidamente } \\
\text { con el tiempo. }\end{array}$ & $\begin{array}{l}\text { Depende de la } \\
\text { proporción de } \\
\text { cada componente. } \\
\text { Aumenta muy } \\
\text { rápidamente con } \\
\text { el tiempo. }\end{array}$ & 3,1 & 1,6 & 0,9 \\
\hline $\begin{array}{l}\text { Tiempo de curado (h) a } \\
25^{\circ} \mathrm{C} \text {, hasta alcanzar la } \\
\text { dureza final y finalizar la } \\
\text { liberación de gases }\end{array}$ & 48 & $24-36$ & $24-36$ & $24-36$ & $24-36$ \\
\hline $\begin{array}{c}\text { Dureza alcanzada des- } \\
\text { pués del curado [HLD] } \\
\text { (error: } 5 \%)\end{array}$ & 787 & 829 & 807 & 799 & 198 \\
\hline $\begin{array}{l}\text { Calidad superficial (ver } \\
\text { Figuras } 4 \text { y 5) }\end{array}$ & Lisa & $\begin{array}{l}\text { Bastante lisa, con } \\
\text { presencia de cris- } \\
\text { talitos. }\end{array}$ & Lisa & $\begin{array}{l}\text { Lisa, pero se } \\
\text { degrada en } \\
\text { ambientes muy } \\
\text { húmedos: se for- } \\
\text { man cráteres }\end{array}$ & $\begin{array}{l}\text { Lisa, pero se } \\
\text { degrada en am- } \\
\text { bientes muy hú- } \\
\text { medos: se torna } \\
\text { pegajosa }\end{array}$ \\
\hline $\begin{array}{l}\text { Predisposición a la for- } \\
\text { mación de } \\
\text { burbujas } \\
\text { (ver Figura 5) }\end{array}$ & $\begin{array}{c}\text { Burbujas y } \\
\text { huecos grandes } \\
\text { (debido a la alta } \\
\text { viscosidad de la } \\
\text { muestra) }\end{array}$ & $\begin{array}{l}\text { Varias burbujas } \\
\text { pequeñas }\end{array}$ & $\begin{array}{c}\text { Muchas burbu- } \\
\text { jas pequeñas }\end{array}$ & $\begin{array}{l}\text { Muy pocas bur- } \\
\text { bujas pequeñas }\end{array}$ & $\begin{array}{l}\text { Muy pocas bur- } \\
\text { bujas pequeñas }\end{array}$ \\
\hline
\end{tabular}




\begin{tabular}{|c|c|c|c|c|c|}
\hline $\begin{array}{l}\text { Respuesta al pulido me- } \\
\text { cánico } \\
\text { (ver Figura 6) }\end{array}$ & $\begin{array}{l}\text { Se pule fácil- } \\
\text { mente, mantie- } \\
\text { ne adherencia }\end{array}$ & $\begin{array}{l}\text { Se pule fácilmen- } \\
\text { te, mantiene adhe- } \\
\text { rencia }\end{array}$ & $\begin{array}{l}\text { Se pule fácil- } \\
\text { mente, mantie- } \\
\text { ne adherencia }\end{array}$ & $\begin{array}{l}\text { Se pule fácil- } \\
\text { mente, mantiene } \\
\text { adherencia }\end{array}$ & $\begin{array}{l}\text { No se pule bien, } \\
\text { pierde adherencia } \\
\text { con el vidrio cal- } \\
\text { cogenuro }\end{array}$ \\
\hline $\begin{array}{c}\text { Efecto sobre pintura de } \\
\text { Ag seca } \\
\text { (ver Figura 7) }\end{array}$ & $\begin{array}{l}\text { Sin efectos } \\
\text { apreciables }\end{array}$ & $\begin{array}{l}\text { Disuelve y corre } \\
\text { la pintura de } \mathrm{Ag}\end{array}$ & $\begin{array}{l}\text { Sin efectos } \\
\text { apreciables }\end{array}$ & $\begin{array}{l}\text { Sin efectos apre- } \\
\text { ciables }\end{array}$ & $\begin{array}{l}\text { Sin efectos apre- } \\
\text { ciables }\end{array}$ \\
\hline $\begin{array}{c}\text { Comportamiento frente a } \\
\text { la inmersión en agua } \\
(8 \text { días }) \\
\text { (ver Figura } 8)\end{array}$ & $\begin{array}{c}\text { Absorbe agua y } \\
\text { se ablanda }\end{array}$ & $\begin{array}{l}\text { Sin efectos apre- } \\
\text { ciables }\end{array}$ & $\begin{array}{l}\text { Sin efectos } \\
\text { apreciables }\end{array}$ & $\begin{array}{l}\text { Sin efectos apre- } \\
\text { ciables }\end{array}$ & $\begin{array}{l}\text { Absorbe agua y } \\
\text { se disuelve lenta- } \\
\text { mente }\end{array}$ \\
\hline $\begin{array}{c}\text { Comportamiento frente a } \\
\text { la inmersión en etanol } \\
(1 \text { día }) \\
\text { (ver Figura } 9)\end{array}$ & $\begin{array}{c}\text { Aumenta su } \\
\text { masa en un } 0,07 \\
\% . \\
\text { Se torna go- } \\
\text { moso, más } \\
\text { amarillento y se } \\
\text { despedaza } \\
\end{array}$ & $\begin{array}{l}\text { Se torna de color } \\
\text { salmón }\end{array}$ & $\begin{array}{l}\text { Ningún efecto } \\
\text { visible }\end{array}$ & $\begin{array}{l}\text { Ningún efecto } \\
\text { visible }\end{array}$ & $\begin{array}{l}\text { Aumenta su masa } \\
\text { en un } 0,22 \% \text {. } \\
\text { Se torna elástico } \\
\text { (como la pelota } \\
\text { saltarina o bola } \\
\text { rebotante) }\end{array}$ \\
\hline $\begin{array}{c}\text { Comportamiento frente a } \\
\text { la inmersión en acetona } \\
(1 \text { día }) \\
(\text { ver Figura } 10)\end{array}$ & $\begin{array}{c}\text { La superficie se } \\
\text { torna gomosa, } \\
\text { mientras que el } \\
\text { interior perma- } \\
\text { nece duro } \\
\end{array}$ & $\begin{array}{l}\text { Se torna de color } \\
\text { salmón, muy go- } \\
\text { moso, pierde la } \\
\text { forma }\end{array}$ & $\begin{array}{l}\text { La superficie se } \\
\text { torna gomosa, } \\
\text { mientras que el } \\
\text { interior perma- } \\
\text { nece duro } \\
\end{array}$ & $\begin{array}{l}\text { La superficie se } \\
\text { torna gomosa, } \\
\text { mientras que el } \\
\text { interior permane- } \\
\text { ce duro } \\
\end{array}$ & $\begin{array}{l}\text { Se torna elástico } \\
\text { (como la pelota } \\
\text { saltarina o bola } \\
\text { rebotante) }\end{array}$ \\
\hline $\begin{array}{l}\text { Comportamiento frente a } \\
\text { la inmersión en } \mathrm{AgNO}_{3} \\
\left(10^{-2} \mathrm{M}\right)(1 \text { día }) \\
\text { (ver Figura 11) }\end{array}$ & $\begin{array}{c}\text { Se tiñe la super- } \\
\text { ficie externa de } \\
\text { un color muy } \\
\text { oscuro }\end{array}$ & $\begin{array}{c}\text { Sin efectos apre- } \\
\text { ciables }\end{array}$ & $\begin{array}{l}\text { Sin efectos } \\
\text { apreciables }\end{array}$ & $\begin{array}{l}\text { Se tiñe levemen- } \\
\text { te la superficie } \\
\text { externa, especial- } \\
\text { mente la región } \\
\text { más porosa (zona } \\
\text { del rechupe oca- } \\
\text { sionado durante } \\
\text { el curado) }\end{array}$ & $\begin{array}{l}\text { Sin efectos apre- } \\
\text { ciables }\end{array}$ \\
\hline
\end{tabular}

\section{DISCUSIONES}

A continuación, analizamos la factibilidad de utilizar los materiales estudiados para encapsular membranas selectivas de iones en función de los resultados reportados en la sección anterior.

En la Figura 8 se observa que el Poxipol absorbe una cantidad importante de agua -que es la base de las soluciones a sensar- ablandándose y perdiendo adherencia con la membrana sensible. El color oscuro de su superficie tras la inmersión en $\mathrm{AgNO}_{3}\left(10^{-2} \mathrm{M}\right)$ (Figura 11) demuestra que, además de agua, el material encapsulante absorbe una cantidad importante de iones de la solución. Por estos motivos, descartamos al Poxipol como material encapsulante de ISEs.

El Vaicel presenta varias propiedades ventajosas para encapsular membranas selectivas de iones, tales como su superficie lisa, facilidad de pulido, buena adherencia y baja absorción de agua (Tabla 1). Su limpieza debería realizarse rápidamente y con precaución, ya que los experimentos demuestran que reacciona con el etanol (cambia de color) y la acetona (cambia de color y se disuelve) (Figuras 9 y 10). El hecho de que su viscosidad aumente muy rápidamente durante el autocurado (Tabla 1), lo vuelve ideal para encapsular ISEs con membranas en forma de película delgada, ya que no es necesario utilizar ningún molde contenedor y facilita el proceso de fabricación del ISE. La mayor desventaja es que disuelve parcialmente y corre la pintura de Ag (Figura 7), por lo que el recubrimiento de los contactos eléctricos debe realizarse con sumo cuidado, ya que una conexión eléctrica defectuosa inutilizaría el ISE. Por ello, debería cubrirse la pintura de Ag con un material intermedio de protección, o utilizarse un exceso de pintura de Ag para conectar el alambre metálico con la película conductora (y, además, se debería evitar realizar la colada del Vaicel sobre una superficie inclinada).

El Wilpox APV 3C y el Araldite presentan otras desventajas a tener en consideración. El primero exhibe muchas burbujas pequeñas, pero solo en volumen y no en la superficie (Figuras 4 y 5), por lo que no alteran la rugosidad del encapsulado. Estas burbujas pequeñas podrían eliminarse realizando una desgasificación de la mezcla en vacío como paso previo a la colada. Además, verificamos que la adherencia tanto con el cable metálico como con el vidrio calcogenuros fue buena, incluso durante le pulido mecánico (Figura 6). Por su parte, si bien el Araldite demostró no absorber grandes cantidades de agua al estar sumergido durante hasta 8 días, sí se degradó ligeramente al ser almacenado en un ambiente muy húmedo (se 
formaron cráteres en su superficie, Figura 5). También se coloreó levemente tras la inmersión en $\mathrm{AgNO}_{3}\left(10^{-2} \mathrm{M}\right)$ (Figura 11), principalmente en la zona superior del cilindro, que fue la más rugosa tras el autocurado. Sin embargo, estas desventajas no anulan al Araldite como candidato para encapsular membranas selectivas de iones, especialmente en bulk, si se toman los recaudos de almacenamiento adecuados (como no almacenar el ISE en un ambiente muy húmedo y, si se almacenara en una solución iónica, evitar sumergir la región rugosa del encapsulado).

El Wilpox Doming P-5 experimenta dos procesos en simultáneo durante su inmersión en agua: absorción y disolución. A partir del segundo día inmerso en agua, su masa comienza a decrecer lentamente, debido a que el proceso de disolución es mayor al de absorción (Figura 8 superior). Tras ser retirado del agua, la pérdida de masa se debe a la evaporación del agua absorbida. Este material también reacciona químicamente con etanol y con acetona (utilizados para limpiar contaminación orgánica), alterando sus propiedades (se tornó elástico tras la inmersión en ambos, Tabla 1). Asimismo, observamos que no sirve para incluir materiales que deban ser pulidos, como es el caso de la membrana en bulk, ya que pierde total adherencia al ser sometido a tensiones mecánicas (Figura 6).

\section{CONCLUSIONES}

En este trabajo analizamos las ventajas y limitaciones de cinco materiales encapsulantes disponibles comercialmente en la región latinoamericana para aislar el contacto eléctrico de los electrodos selectivos de iones de contacto sólido con membranas sensibles de vidrios calcogenuros, tanto en bulk como en forma de películas delgadas. Evaluamos su facilidad de conformación, sus propiedades mecánicas, su compatibilidad química con agua (base de las soluciones a sensar), etanol y acetona (comúnmente utilizados para la limpieza de la contaminación orgánica) y con la pintura de Ag seca (utilizada para el contacto eléctrico entre el cable y la membrana sensible).

Para encapsular ISEs con membranas de vidrios calcogenuros en bulk, encontramos que los mejores materiales resultaron ser las resinas epoxi Wilpox APV 3C y Araldite CY-248 + HY-956, teniéndose que tomar algunos recaudos para el almacenamiento en el caso del Araldite CY-248 + HY-956.

Para encapsular ISEs con membranas en forma de películas delgadas, encontramos que los mejores materiales resultaron ser la resina epoxi Wilpox APV 3C y la resina acrílica Vaicel. El único inconveniente del Wilpox APV 3C proviene de la dificultad de mantener contenida a la resina epoxi durante el curado. En cuanto al Vaicel, se deben tomar los cuidados necesarios para evitar fallas en los contactos eléctricos con pintura de Ag y se deben extremar los cuidados en la limpieza si se utilizan alcohol etílico o acetona.

\section{AGRADECIMIENTOS}

Los autores agradecen a Hernán G. Svoboda del Laboratorio de Materiales y Estructuras por los ensayos de dureza. También agradecen el financiamiento de la Universidad de Buenos Aires, del CONICET y de la Agencia Nacional de Promoción Científica y Tecnológica, especialmente en el marco de los proyectos UBACyT 20020130300056BA, 20020170200094BA у 20020170100520BA, PIP-2015-2017-GI-11220150100775CO у PICT 2014-0990 у 2015-1238.

\section{BIBLIOGRAFÍA}

[1] VLASOV, Y.G., BYCHKOV, E.A., BRATOV, A.V., "Ion-selective field-effect transistor and chalcogenide glass ion-selective electrode systems for biological investigations and industrial applications", Analyst, v. 119, n. 3, pp. 449-454, 1994. https://doi.org/10.1039/AN9941900449

[2] MORENO, T.V., MALACARNE, L.C., BAESSO, M.L., QU, W., DY, E., XIE, Z., FAHLMAN, J., SHEN, J., ASTRATH, N.G., "Potentiometric sensors with chalcogenide glasses as sensitive membranes: A short review", Journal of Non-Crystalline Solids, v. 495, pp. 8-18, 2018. https://doi.org/10.1016/j.jnoncrysol.2018.04.057

[3] BRIERLEY, C.L., "Bioremediation of metal contaminated surface and groundwaters", Geomicrobiology Journal, v. 8, n. 3-4, pp. 201-223, 1990. https://doi.org/10.1080/01490459009377894

[4] TRACHTENBERG, I., BAKER, C.T., "Ion-selective electrochemical sensors", Research and Development Progress Report - Office of Saline Water, v. 496, pp. 1-105, 1969. https://hdl.handle.net/2027/mdp.39015078500561

[5] TANG, X., WANG, P.-Y., BUCHTER, G., "Ion-Selective Electrodes for Detection of Lead (II) in Drinking Water: A Mini-Review", Environments, v. 5, n. 9, pp. 95. 91-14, 2018. https://doi.org/10.3390/environments5090095

[6] SCHÖNING, M.J., KLOOCK, J.P., “About 20 years of silicon-based thin-film sensors with chalcogenide glass materials for heavy metal analysis: Technological aspects of fabrication and miniaturization”, Electroanalysis, v. 19, n. 19-20, 
pp. 2029-2038, 2007. https://doi.org/10.1002/elan.200703955

[7] VLASOV, Y., LEGIN, A., RUDNITSKAYA, A., et al. "Nonspecific sensor arrays (" electronic tongue") for chemical analysis of liquids “, Pure and Applied Chemistry, v. 77, n. 11, pp. 1965-1983, 2005. https://doi.org/10.1351/ pac200577111965

[8] FJELDLY, T., NAGY, K., STARK, B., “Solid state differential potentiometric sensors", Sensors and Actuators, v. 3, pp. 111-118, 1982. https://doi.org/10.1016/0250-6874(82)80012-7

[9] HO, N., KRATOCHVIL, J., BLACKBURN, G., et al. "Encapsulation of polymeric membrane-based ion-selective field effect transistors", Sensors and Actuators, v. 4, pp. 413-421, 1983. https://doi.org/10.1016/0250-6874(83)85052-5

[10] MATSUO, T., ESASHI, M., "Methods of ISFET fabrication”, Sensors and Actuators, v. 1, pp. 77-96, 1981. https:// doi.org/10.1016/0250-6874(81)80006-6

[11] VASSILEV, V., HRISTOVA-VASILEVA, T., ALJIHMANI, L., et al."Sn (11)-ion-selective electrode based on chalcogenide glasses”, Faculty of Mathematics \& Natural Sciences, v. 2, pp. 30-36, 2007. https://www.researchgate.net/ profile/L_Aljihmani/publication/262231382_SNII-ION-SELECTIVE_ELECTRODE_BASED_ON_CHALCOGENIDE_GLASSES/links/0f3175371cce13461a000000.pdf

[12] SPIRIDON, O.B., "Cd (II)-selective potentiometric sensor with multilayer solid membrane based on selenide and its applications", Journal of optoelectronics and advanced materials, v. 12, n. 11, pp. 2226-2235, 2010. https://www.researchgate.net/profile/Bizerea_Otilia/publication/286939360_Cd_II-selective_potentiometric_sensor_with_multilayer_solid_membrane_based_on_selenide_and_its_applications/links/590b2d6b458515ebb4a85ce1/Cd-II-selective-potentiometric-sensor-with-multilayer-solid-membrane-based-on-selenide-and-its-applications.pdf

[13] CONDE GARRIDO, J.M., SILVEYRA, J.M., UREÑA, M.A., "Multi-ion and pH sensitivity of AgGeSe ion selective electrodes", Journal of Physics and Chemistry of Solids, v. 89, pp. 115-119, 2016. https://doi.org/10.1016/j. jpcs.2015.10.015

[14] CHEN, L., ZENG, X., HE, X., "Selective electrodes for silver based on polymeric membranes containing calix [4] arene derivatives", Fresenius' Journal of Analytical Chemistry, v. 367, n. 6, pp. 535-538, 2000. https://doi.org/10.1007/ s002160000397

\section{ORCID}

Josefina María Silveyra María Andrea Ureña Juan Manuel Conde Garrido https://orcid.org/0000-0003-0307-3419

https://orcid.org/0000-0001-8900-5243

https://orcid.org/0000-0002-7689-3912 